\title{
The disturbance decoupling problem with measurement feedback and stability for systems with direct feedthrough matrices
}

\author{
A.A. Stoorvogel * \\ Dept. of Electrical Engineering and Computer Science, University of Michigan, Ann Arbor, MI, USA \\ J.W. van der Woude \\ Dept. of Technical Mathematics and Informatics, Delft University of Technology, Delft, Netherlands
}

Received 13 December 1990

Revised 6 May 1991

Abstract: In this paper the known geometric results on the disturbance decoupling problem with measurement feedback and internal stability (DDPMS) are extended to include non-zero direct feedthrough matrices. Necessary and sufficient conditions for the solvability of the DDPMS are expressed in terms of three subspace inclusions.

Keywords: Disturbance decoupling; geometric approach; measurement feedback.

\section{Introduction}

The so-called disturbance decoupling problems have been investigated extensively in the last two decades. It was the starting point for the development of a geometric approach to systems theory. The problem is to find a compensator such that the closed loop transfer matrix from disturbance to output is equal to 0 . Using the concept of $(A, B)$-invariance, the disturbance decoupling problem with state feedback (DDP) was solved in $[2,3,21]$. The problem of disturbance decoupling with state feedback and the extra requirement of internal stability (DDPS), was solved in [6,21]. A detailed reference for the above mentioned problems is [22]. Approximately 10 years later, the above mentioned problems were solved for the case of measurement feedback. The disturbance decoupling problem with measurement feedback (DDPM) was solved in [1,7]. Finally, the disturbance decoupling problem with measurement feedback and internal stability was solved in $[5,18]$.

All the above problems have also been generalized to the so-called almost disturbance decoupling problems where one investigates under which conditions we can make the closed loop transfer matrix arbitrarily small in a suitable norm. References for these generalizations are $[14,19,20]$. For a overview of all the existing results we refer to [15].

In all of the above references the direct feedthrough matrices of the system are assumed to be equal to zero. In the state space approach to $H_{\infty}$ and LQG control however disturbance decoupling problems for systems with direct feedthrough matrices do play a role (see $[11,12])$. Therefore, this paper will extend the results on the disturbance decoupling problems mentioned above to include direct feedthrough matrices. We will solve the problem of disturbance decoupling with measurement feedback and internal stability (DDPMS) with respect to some arbitrary stability set. Results for DDP, DDPS and DDPM follow as special cases from our main theorem. Note that an extension of the results on almost disturbance

* On leave from the Dept. of Mathematics and Computing Science, Eindhoven University of Technology, Netherlands. 
decoupling to incorporate direct feedthrough matrices has already been done in [23].

We will use a geometric approach in this paper similar to the techniques used in the above mentioned references. We find that solvability of the DDPMS is equivalent to the requirement that three subspace inclusions hold. We will derive our results for continuous time systems but the related results for discrete time systems are identical.

In this paper we will present our main result in Section 2. In Section 3 we will prove our main result. In Section 4 we will discuss some extensions. Finally we conclude with Section 5 which contains some concluding remarks.

\section{Problem formulation and results}

Consider the finite-dimensional linear time-invariant system $\Sigma$ given by

$$
\Sigma:\left\{\begin{array}{l}
\dot{x}=A x+B u+E d, \\
y=C_{1} x+D_{1} d, \\
z=C_{2} x+D_{2} u,
\end{array}\right.
$$

where $x \in \mathbb{R}^{n}$ is the state of the system, $u \in \mathbb{R}^{m}$ the control input, $d \in \mathbb{R}^{q}$ the disturbance input, $y \in \mathbb{R}^{p}$ the measurement output and $z \in \mathbb{R}^{r}$ the output of $\Sigma$ to be controlled. $A, B, E, C_{1}, C_{2}, D_{1}$ and $D_{2}$ are real matrices of appropriate dimensions.

Assume that system (2.1) is controlled by means of a measurement feedback compensator $\Sigma_{F}$ described by

$$
\Sigma_{F}:\left\{\begin{array}{l}
\dot{p}=K p+L y, \\
u=M p+N y,
\end{array}\right.
$$

with $p \in \mathbb{R}^{k}$ the state of the compensator and $K, L, M$ and $N$ real matrices of appropriate dimensions. Interconnection of $\Sigma$ and $\Sigma_{F}$ results in a closed loop system $\Sigma_{\mathrm{cl}}=\Sigma \times \Sigma_{F}$ described by

$$
\Sigma_{\mathrm{cl}}:\left\{\begin{array}{l}
\dot{x}_{\mathrm{e}}=A_{\mathrm{e}} x_{\mathrm{e}}+B_{\mathrm{e}} d, \\
z=C_{\mathrm{e}} x_{\mathrm{e}}+D_{\mathrm{e}} d
\end{array}\right.
$$

where we have denoted

$$
\begin{aligned}
& x_{\mathrm{e}}=\left(\begin{array}{c}
x \\
p
\end{array}\right), \quad A_{\mathrm{e}}=\left(\begin{array}{cc}
A+B N C_{1} & B M \\
L C_{1} & K
\end{array}\right), \quad B_{\mathrm{e}}=\left(\begin{array}{c}
E+B N D_{1} \\
L D_{1}
\end{array}\right), \\
& C_{\mathrm{e}}=\left(C_{2}+D_{2} N C_{1} \quad D_{2} M\right), \quad D_{\mathrm{e}}=D_{2} N D_{1} .
\end{aligned}
$$

Let $C_{g}$ be a subset of the complex plane $C$ which is symmetric $\left(\lambda \in \mathbb{C}_{g} \Leftrightarrow \bar{\lambda} \in \mathrm{C}_{g}\right)$ and with at least one point on the real axis $\left(\mathbb{C}_{g} \cap \mathbf{R} \neq \emptyset\right)$. The interconnection of a system $\Sigma$ and $\Sigma_{F}$ as given in (2.3) is called $\mathbf{C}_{g}$-stable if the matrix $A_{\mathrm{e}}$ is $\mathbb{C}_{g}$-stable, i.e. all eigenvalues of $A_{\mathrm{e}}$ are contained in $\mathbb{C}_{g}$.

We can now consider the following problem:

Problem. Given $\Sigma$ determine $\Sigma_{F}$ such that the interconnection $\Sigma \times \Sigma_{F}$ is $\mathbf{C}_{g}$-stable and such that the closed loop transfer matrix is equal to zero.

This problem is often called the disturbance decoupling problem with measurement feedback and internal stability (DDPMS). The problem has been completely solved in case the direct feedthrough matrices $D_{1}$ and $D_{2}$ are equal to 0 (see $[4,9,18]$ ). This paper extends these results to include direct feedthrough matrices.

For the formulation of our main result we need a number of definitions: 
Definition 2.1. We define the detectable strongly controllable subspace $\mathscr{T}_{g}(A, B, C, D)$ as the smallest subspace $\mathscr{T}$ of $\mathbb{R}^{n}$ for which there exists a linear mapping $G$ such that the following subspace inclusions are satisfied:

$$
\begin{aligned}
& (A+G C) \mathscr{T} \subseteq \mathscr{T}, \\
& \operatorname{Im}(B+G D) \subseteq \mathscr{T},
\end{aligned}
$$

and such that $A+G C \mid \mathbb{R}^{n} / \mathscr{T}$ is $\mathbf{C}_{g}$-stable. We also define the stabilizable weakly unobservable subspace $\mathscr{V}_{g}(A, B, C, D)$ as the largest subspace $\mathscr{V}$ for which there exists a mapping $F$ such that the following subspace inclusions are satisfied:

$$
\begin{aligned}
& (A+B F) \mathscr{V} \subseteq \mathscr{V}, \\
& (C+D F) \mathscr{V}=\{0\},
\end{aligned}
$$

and such that $A+B F \mid \mathscr{V}$ is $\mathbb{C}_{g}$-stable.

The subspaces $\mathscr{V}_{g}(A, B, C, D)$ and $\mathscr{T}_{g}(A, B, C, D)$ can be computed by means of well-known algorithms (see e.g. [10,22]). We also note that if $(A, B)$ is $\mathbf{C}_{g}$-stabilizable then for $\mathscr{V}_{g}(A, B, C, D)$ there exists an $F$ such that (2.6) and (2.7) are satisfied and moreover $A+B F$ is $\mathbf{C}_{g}$-stable. A similar comment can be made for $\mathscr{T}_{g}(A, B, C, D)$ in case $(C, A)$ is $\mathbf{C}_{g}$-detectable. We will denote, for a given subspace $\mathscr{L}$ and matrix $C$, by $C^{-1} \mathscr{L}$ the subspace $\{x \mid C x \in \mathscr{L}\}$.

We can now formulate the main result from this paper:

Theorem 2.2. Let $\Sigma$ be given of the form (2.1). The following two conditions are equivalent:

(i) There exists a compensator of the form (2.2) such that the closed loop system is $\mathbf{C}_{g}$-stable and such that the closed loop transfer matrix is equal to 0.

(ii) $(A, B)$ is $\mathbb{C}_{g^{-s t a b i l i z a b l e}}\left(C_{1}, A\right)$ is $\mathbb{C}_{g^{-} \text {detectable and }}$

(a) $\operatorname{Im} E \subseteq \mathscr{V}_{g}\left(\Sigma_{\mathrm{ci}}\right)+B \operatorname{Ker} D_{2}$,

(b) $\operatorname{Ker} C_{2} \supseteq \mathscr{T}_{g}\left(\Sigma_{\mathrm{di}}\right) \cap C_{1}^{-1} \operatorname{Im} D_{1}$,

(c) $\mathscr{T}_{g}\left(\Sigma_{d i}\right) \subseteq \mathscr{V}_{g}\left(\Sigma_{\mathrm{ci}}\right)$

where $\Sigma_{\mathrm{ci}}=\left(A, B, C_{2}, D_{2}\right)$ and $\Sigma_{\mathrm{di}}=\left(A, E, C_{1}, D_{1}\right)$.

Remarks. (1) Note that we have still all freedom in our choice of $\mathbf{C}_{\boldsymbol{g}}$. For the disturbance decoupling problem with measurement feedback (DDPM) we choose $\mathbb{C}_{g}=\mathbf{C}$. On the other hand if we are interested in disturbance decoupling with internal (asymptotic) stability, then we choose $\mathbf{C}_{g}$ equal to the open left half complex plane.

(2) Since we mainly investigate properties of transfer matrices, the results for discrete time systems are immediate from Theorem 2.2. For a discrete time system with the same parameters as $\Sigma$ conditions (i) and (ii) are still equivalent. Only this time, for internal (asymptotic) stability, we have to choose $\mathbb{C}_{g}$ equal to the open unit disc.

(3) In the special case of state feedback, i.e. $C_{1}=I$ and $D_{1}=0$, conditions (a)-(c) in our theorem reduce to:

(a) $\operatorname{Im} E \subseteq \mathscr{V}_{g}\left(\Sigma_{\mathrm{ci}}\right)+B \operatorname{Ker} D_{2}$

(b) $\operatorname{Ker} C_{2} \supseteq\{0\}$,

(c) $\operatorname{Im} E \subseteq \mathscr{V}_{g}\left(\Sigma_{\mathrm{ci}}\right)$

respectively. Clearly condition (c) implies conditions (a) and (b). Thus we obtain the well-known single subspace inclusion $\operatorname{Im} E \subseteq \mathscr{\mathscr { V }}_{g}\left(\Sigma_{\mathrm{ci}}\right)$ for this special case.

(4) It can be easily checked that condition (c) also implies conditions (a) and (b) in case both the direct feedthrough matrices $D_{1}$ and $D_{2}$ are equal to 0 .

The following theorem gives the possibility of actually calculating a suitable compensator if it exists: 
Theorem 2.3. Let $\Sigma$ of the form (2.1) be given. Conditions $(a)-(c)$ of Theorem 2.2 are equivalent to the following conditions: $\mathscr{T}_{\mathrm{g}}\left(\Sigma_{\mathrm{di}}\right) \subseteq \mathscr{V}_{g}\left(\Sigma_{\mathrm{ci}}\right)$ and there is a matrix $N$ such that

$$
\left(\left[\begin{array}{ll}
A & E \\
C_{2} & 0
\end{array}\right]+\left[\begin{array}{c}
B \\
D_{2}
\end{array}\right] N\left[\begin{array}{ll}
C_{1} & D_{1}
\end{array}\right]\right)\left(\mathscr{T}_{g}\left(\Sigma_{\mathrm{di}}\right) \oplus \mathbb{R}^{q}\right) \subseteq\left(\mathscr{V}_{g}\left(\Sigma_{\mathrm{ci}}\right) \oplus\{0\}\right) .
$$

In addition to such an $N$, let $F$ and $G$ be the matrices satisfying the conditions of Definition 2.1 for $\mathscr{V}_{g}\left(\Sigma_{\mathrm{ci}}\right)$ and $\mathscr{T}_{g}\left(\Sigma_{\mathrm{di}}\right)$ respectively and such that $A+B F$ and $A+G C_{1}$ are both $\mathbb{C}_{g}$-stable. Then a compensator $\Sigma_{F}$ making the closed loop system $\mathbb{C}_{g}$-stable and the closed loop transfer matrix equal to 0 is given by

$$
\Sigma_{F}:\left\{\begin{array}{l}
\dot{p}=A p+B u+G\left(C_{1} p-y\right), \\
u=F p-N\left(C_{1} p-y\right) .
\end{array}\right.
$$

Remarks. (1) In case $\mathbb{C}_{g}=\mathbb{C}$, i.e. if we consider disturbance decoupling without stability requirements, and if the conditions (ii) of Theorem 2.2 are satisfied then it can be shown that there exists a compensator of dynamic order:

$$
\operatorname{dim} \mathscr{V}_{g}\left(\Sigma_{\mathrm{ci}}\right)-\operatorname{dim} \mathscr{T}_{g}\left(\Sigma_{\mathrm{di}}\right)
$$

This result was already known (see [9]) and can be extended to the more general system (2.1) investigated in this paper. By means of a simple example it can be shown that this result is not true in case of stability requirements $\left(\mathbb{C}_{g} \neq \mathbf{C}\right)$. For bounds on the order of the compensator that do hold in case $\mathbb{C}_{g} \neq \mathbb{C}$ we refer to [13].

(2) Note that we can easily rewrite (2.9) in the form (2.2). However in (2.9) the structure of the controller is more visible.

We can also investigate when there exists a strictly proper compensator which solves th DDPMS:

Theorem 2.4. Let $\Sigma$ be given of the form (2.1). The following two conditions are equivalent:

(i) There exists a compensator of the form (2.2) with $N=0$ such that the closed loop system is $\mathbb{C}_{g}$-stable and such that the closed loop transfer matrix is equal to 0.

(ii) $(A, B)$ is $\mathbb{C}_{\mathrm{g}}$-stabilizable, $\left(C_{1}, A\right)$ is $\mathbb{C}_{\mathrm{g}}$-detectable and
(a) $\operatorname{Im} E \subseteq \mathscr{V}_{g}\left(\Sigma_{\text {ci }}\right)$,
(b) $\operatorname{Ker} C_{2} \supseteq \mathscr{T}_{\mathrm{g}}\left(\Sigma_{\mathrm{di}}\right)$
(c) $A \mathscr{T}_{\mathrm{g}}\left(\Sigma_{\mathrm{di}}\right) \subseteq \mathscr{V}_{\mathrm{g}}\left(\Sigma_{\mathrm{ci}}\right)$
(d) $\mathscr{T}_{\mathrm{g}}\left(\Sigma_{\mathrm{di}}\right) \subseteq \mathscr{V}_{\mathrm{g}}\left(\Sigma_{\mathrm{ci}}\right)$

where $\Sigma_{\mathrm{ci}}=\left(A ; B, C_{2}, D_{2}\right)$ and $\Sigma_{\mathrm{di}}=\left(A, E, C_{1}, D_{1}\right)$.

In case part (ii) is satisfied a compensator satisfying (i) is given by (2.9) with $N=0$ and $F, G$ as described in Theorem 2.3.

In the next section the above results will be proven.

\section{Proofs of the results obtained}

The following characterization (see e.g. [4]) of the subspace $\mathscr{V}_{g}(\Sigma)$ turns out to be very useful:

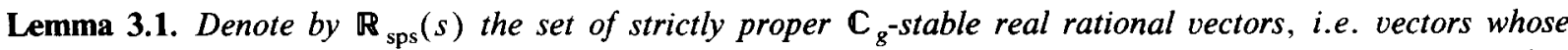
elements are strictly proper rational functions with all poles in $\mathbf{C}_{g}$. The subspace $\mathscr{V}_{g}(A, B, C, D)$ is equal to the set of all $x_{0} \in \mathbb{R}^{n}$ for which there exist $\xi, \omega \in \mathbb{R}_{\text {sps }}(s)$ such that

$$
x_{0}=(s I-A) \xi(s)-B \omega(s), \quad 0=C \xi(s)+D \omega(s) .
$$


We will also need the following lemma.

Lemma 3.2. Let $\mathscr{U}$ and $\mathscr{W}$ be linear subspaces, and let $P, Q$ and $R$ be matrices such that $P \mathscr{U} \subseteq \mathscr{W}+\operatorname{Im} Q, \quad P(\mathscr{U} \cap \operatorname{Ker} R) \subseteq \mathscr{W}$.

Then there is a matrix $X$ such that

$$
(P+Q X R) \mathscr{U} \subseteq \mathscr{W} .
$$

A proof of the above lemma is straightforward and goes along the same lines as the proof of Lemma 4.2 in [8].

Proof of Theorem 2.2. (ii) $\Rightarrow$ (i): Because $(A, B)$ and $\left(C_{1}, A\right)$ are $\mathbb{C}_{g}$-stabilizable and $\mathbf{C}_{g}$-detectable respectively it follows from the definitions of $\mathscr{V}_{g}\left(\Sigma_{\mathrm{ci}}\right)$ and $\mathscr{T}_{g}\left(\Sigma_{\mathrm{di}}\right)$ that there are matrices $F$ and $G$ such that the subspace inclusions in Definition 2.1 with $\mathscr{V}=\mathscr{V}_{g}\left(\Sigma_{\mathrm{ci}}\right)$ and $\mathscr{T}=\mathscr{T}_{g}\left(\Sigma_{\mathrm{di}}\right)$ are satisfied, and such that $A+B F$ and $A+G C_{1}$ are both $\mathrm{C}_{g}$-stable.

Now observe that $\mathscr{V}_{g}\left(\Sigma_{\text {ci }}\right)$ and $\mathscr{T}_{g}\left(\Sigma_{\text {di }}\right)$ are such that

$$
\begin{aligned}
& {\left[\begin{array}{l}
A \\
C_{2}
\end{array}\right] \mathscr{V}_{g}\left(\Sigma_{\mathrm{ci}}\right) \subseteq\left(\mathscr{V}_{g}\left(\Sigma_{\mathrm{ci}}\right) \oplus\{0\}\right)+\operatorname{Im}\left[\begin{array}{c}
B \\
D_{2}
\end{array}\right],} \\
& {\left[\begin{array}{ll}
A & E
\end{array}\right]\left(\left(\mathscr{T}_{g}\left(\Sigma_{\mathrm{di}}\right) \oplus \mathbb{R}^{q}\right) \cap \operatorname{Ker}\left[\begin{array}{ll}
C_{1} & D_{1}
\end{array}\right]\right) \subseteq \mathscr{T}_{g}\left(\Sigma_{\mathrm{di}}\right) .}
\end{aligned}
$$

Furthermore, it can be easily shown that condition (a) is equivalent to

$$
\operatorname{Im}\left[\begin{array}{c}
E \\
0
\end{array}\right] \subseteq\left(\mathscr{V}_{g}\left(\Sigma_{\mathrm{ci}}\right) \oplus\{0\}\right)+\operatorname{Im}\left[\begin{array}{c}
B \\
D_{2}
\end{array}\right],
$$

and that condition (b) is equivalent to

$$
\left(\mathscr{T}_{g}\left(\Sigma_{\mathrm{di}}\right) \oplus \mathbb{R}^{q}\right) \cap \operatorname{Ker}\left[C_{1} \quad D_{1}\right] \subseteq \operatorname{Ker}\left[\begin{array}{ll}
C_{2} & 0
\end{array}\right] .
$$

Now, using condition (c), the combination of (3.1) and (3.3) implies that

$$
\left[\begin{array}{ll}
A & E \\
C_{2} & 0
\end{array}\right]\left(\mathscr{T}_{g}\left(\Sigma_{\mathrm{di}}\right) \oplus \mathbb{R}^{q}\right) \subseteq\left(\mathscr{V}_{g}\left(\Sigma_{\mathrm{ci}}\right) \oplus\{0\}\right)+\operatorname{Im}\left[\begin{array}{c}
B \\
D_{2}
\end{array}\right],
$$

while the combination of (3.2) and (3.4) with condition (c) implies that

$$
\left[\begin{array}{cc}
A & E \\
C_{2} & 0
\end{array}\right]\left(\left(\mathscr{T}_{g}\left(\Sigma_{\mathrm{di}}\right) \oplus \mathbb{R}^{q}\right) \cap \operatorname{Ker}\left[C_{1} \quad D_{1}\right]\right) \subseteq\left(\mathscr{V}_{g}\left(\Sigma_{\mathrm{ci}}\right) \oplus\{0\}\right) .
$$

The last two inclusions imply by Lemma 3.2 the existence of a matrix $N$ satisfying (2.8), or equivalently such that

$$
\begin{aligned}
& \left(A+B N C_{1}\right) \mathscr{T}_{g}\left(\Sigma_{\mathrm{di}}\right) \subseteq \mathscr{V}_{g}\left(\Sigma_{\mathrm{ci}}\right), \\
& \left(C_{2}+D_{2} N C_{1}\right) \mathscr{T}_{g}\left(\Sigma_{\mathrm{di}}\right)=\{0\}, \quad D_{2} N D_{1}=0 .
\end{aligned}
$$

Let such $N$ be the feedthrough matrix of the compensator (2.2), and define the other compensator matrices as follows:

$$
K:=A+B F+G C_{1}-B N C_{1}, \quad L:=B N-G, \quad M:=F-N C_{1} .
$$

It is easy to see that the closed loop system now obtained can equivalently be described as follows:

$$
\Sigma_{\mathrm{cl}}:\left\{\begin{array}{l}
\left(\begin{array}{c}
\dot{x} \\
\dot{x}-\dot{p}
\end{array}\right)=\left(\begin{array}{cc}
A+B F & B N C_{1}-B F \\
0 & A+G C_{1}
\end{array}\right)\left(\begin{array}{c}
x \\
x-p
\end{array}\right)+\left(\begin{array}{c}
E+B N D_{1} \\
E+G D_{1}
\end{array}\right) d, \\
z=\left(\begin{array}{ll}
C_{2}+D_{2} F & D_{2} N C_{1}-D_{2} F
\end{array}\right)\left(\begin{array}{c}
x-p \\
x-p
\end{array}\right)+D_{2} N D_{1} d .
\end{array}\right.
$$


An easy calculation shows that the closed loop transfer matrix is given by

$$
\begin{aligned}
& \left(C_{2}+D_{2} F\right)(s I-A-B F)^{-1}\left(E+B N D_{1}\right) \\
& \quad-\left(C_{2}+D_{2} F\right)(s I-A-B F)^{-1}\left(s I-A-B N C_{1}\right)\left(s I-A-G C_{1}\right)^{-1}\left(E+G D_{1}\right) \\
& \quad+\left(C_{2}+D_{2} N C_{1}\right)\left(s I-A-G C_{1}\right)^{-1}\left(E+G D_{1}\right)+D_{2} N D_{1} .
\end{aligned}
$$

From (3.6) it is clear that the closed loop system is $\mathbb{C}_{g}$ stable, since $A+B F$ and $A+G C_{1}$ are $\mathbb{C}_{g}$-stable. Moreover observe that $F$ and $G$ are such that for all $s \in \mathbf{C}$,

$$
\begin{aligned}
& \operatorname{Ker}\left(C_{2}+D_{2} F\right)(s I-A-B F)^{-1} \supseteq \mathscr{V}_{g}\left(\Sigma_{\mathrm{ci}}\right), \\
& \operatorname{Im}\left(s I-A-G C_{1}\right)^{-1}\left(E+G D_{1}\right) \subseteq \mathscr{T}_{g}\left(\Sigma_{\mathrm{di}}\right) .
\end{aligned}
$$

Using (3.5), (3.7) and (3.8) together with condition (c) of Theorem 2.2 it is straightforward to show that the closed loop transfer matrix is equal to 0 .

(i) $\Rightarrow$ (ii): Let a compensator $\Sigma_{F}$ be given such that the closed loop system $\Sigma_{\mathrm{cl}}$ is $C_{g}$-stable and such that the closed loop transfer matrix is equal to 0 , and let the closed loop system $\Sigma_{\text {cl }}$ be described by (2.3). Then all eigenvalues of $A_{\mathrm{e}}$ are in $\mathbb{C}_{g}$ and

$$
C_{\mathrm{e}}\left(s I-A_{\mathrm{c}}\right)^{-1} B_{\mathrm{c}}+D_{\mathrm{e}}=0 .
$$

By the fact that $A_{\mathrm{e}}$ is $\mathbb{C}_{g}$-stable, it is immediate that $(A, B)$ must be $\mathbb{C}_{g}$-stabilizable and $\left(C_{1}, A\right)$ $C_{g}$-detectable. Because the closed loop transfer matrix is zero, it follows that $D_{\mathrm{e}}=0$. We define $\mathscr{V}_{\mathrm{e}}:=\left\langle A_{\mathrm{e}} \mid \operatorname{Im} B_{\mathrm{e}}\right\rangle$, i.e. $\mathscr{V}_{\mathrm{e}}$ is the smallest $A_{\mathrm{e}}$-invariant subspace containing $\operatorname{Im} B_{\mathrm{e}}$. Since $C_{\mathrm{e}}\left(s I-A_{\mathrm{e}}\right)^{-1} B_{\mathrm{e}}=$ 0 , the definition of $\mathscr{V}_{\mathrm{e}}$ implies that $\mathscr{V}_{\mathrm{e}} \subseteq \operatorname{Ker} C_{\mathrm{e}}$. Define

$$
\begin{aligned}
& \mathscr{T}:=\left\{x \in \mathbb{R}^{n} \mid\left(\begin{array}{l}
x \\
0
\end{array}\right) \in \mathscr{V}_{\mathrm{e}}\right\}, \\
& \mathscr{V}:=\left\{x \in \mathbb{R}^{n} \mid \exists p \in \mathbb{R}^{k}:\left(\begin{array}{l}
x \\
p
\end{array}\right) \in \mathscr{V}_{\mathrm{e}}\right\} .
\end{aligned}
$$

Clearly $\mathscr{T} \subseteq \mathscr{V}$. Moreover, it follows that

$$
\begin{aligned}
& \left(A+B N C_{1}\right) \mathscr{T} \subseteq \mathscr{V}, \quad \operatorname{Im}\left(E+B N D_{1}\right) \subseteq \mathscr{V}, \\
& \left(C_{2}+D_{2} N C_{1}\right) \mathscr{T}=\{0\}, \quad D_{2} N D_{1}=0 .
\end{aligned}
$$

Take any $x \in \mathscr{V}$. By definition of $\mathscr{V}$ there exists $p \in \mathbb{R}^{k}$ such that $\left(x^{\mathrm{T}} p^{\mathrm{T}}\right)^{\mathrm{T}} \in \mathscr{V}_{\mathrm{e}}$. Define

$$
\left(\begin{array}{c}
\xi(s) \\
\omega(s)
\end{array}\right):=\left(s I-A_{\mathrm{e}}\right)^{-1}\left(\begin{array}{l}
x \\
p
\end{array}\right) .
$$

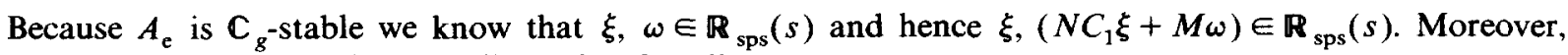
because $\mathscr{V}_{\mathrm{e}}$ is $A_{\mathrm{e}}$-invariant it follows that for all $s$,

$$
\left(\begin{array}{c}
\xi(s) \\
\omega(s)
\end{array}\right) \in \mathscr{V}_{\mathrm{e}} \subseteq \operatorname{Ker} C_{\mathrm{e}} .
$$

Combining the above, we find that

$$
\begin{aligned}
& x=(s I-A) \xi(s)-B\left(N C_{1} \xi(s)+M \omega(s)\right), \\
& 0=C_{2} \xi(s)+D_{2}\left(N C_{1} \xi(s)+M \omega(s)\right)
\end{aligned}
$$


This implies by Lemma 3.1 that $x \in \mathscr{V}_{g}\left(\Sigma_{\mathrm{ci}}\right)$. Hence $\mathscr{V} \subseteq \mathscr{V}_{g}\left(\Sigma_{\mathrm{ci}}\right)$. By dual reasoning it can be derived that $\mathscr{T}_{\mathrm{g}}\left(\Sigma_{\mathrm{di}}\right) \subseteq \mathscr{T}$. From the above it is clear that condition (c) of (ii) is satisfied. From (3.11) it is now clear that

$$
\begin{aligned}
& \operatorname{Im}\left(E+B N D_{1}\right) \subseteq \mathscr{V}_{g}\left(\Sigma_{\mathrm{ci}}\right), \\
& \operatorname{Ker}\left(C_{2}+D_{2} N C_{1}\right) \supseteq \mathscr{T}_{g}\left(\Sigma_{\mathrm{di}}\right), \\
& D_{2} N D_{1}=0 .
\end{aligned}
$$

Now (3.12) and (3.14) together imply condition (a) of (ii). Indeed take any $x \in \mathbb{R}^{q}$ and denote $u=N D_{1} x$. Then $u \in \operatorname{Ker} D_{2}$ and there is a $v \in \mathscr{V}_{g}\left(\Sigma_{\text {ci }}\right)$ such that $E x=v-B u$. Dually it can be shown that (3.13), (3.14) together imply condition (b) of (ii).

Theorems 2.3 and 2.4 immediately follow from the proof of Theorem 2.2 .

\section{A more general case}

In Section 2 we discussed a system $\Sigma$ of the form (2.1). However, in the most general linear, time-invariant case, there are two more direct feedthrough matrices unequal to 0 in the system. In this section we discuss this more general case and we assume that our system is of the form

$$
\tilde{\Sigma}:\left\{\begin{array}{l}
\dot{x}=A x+B u+E d \\
y=C_{1} x+D_{3} u+D_{1} d \\
z=C_{2} x+D_{2} u+D_{4} d
\end{array}\right.
$$

For this more general case we have to discuss the admissibility of controllers of the form (2.2) in more detail. Consider the interconnection shown in Figure 1 where $\tilde{\Sigma}$ and $\Sigma_{F}$ are described by (4.1) and (2.2) respectively.

The closed loop system is said to be well-defined and 'internally proper' if the closed loop transfer matrices from $d, v_{1}, v_{2}$ to $z, u, y$ are well-defined and proper. In that case we call the interconnection well-posed. For a detailed discussion of the concept of well-posedness we refer to [17]. It can be shown that here well-posedness is equivalent to the requirement that $I-D_{3} N$ is invertible. Therefore we require that our controller $\Sigma_{F}$ is such that $I-N D_{3}$ is invertible. Moreover, if $I-N D_{3}$ is invertible, the closed loop system $\tilde{\Sigma} \times \Sigma_{F}$ can be written in the form (2.3) where

$$
A_{\mathrm{e}}:=\left(\begin{array}{cc}
A+B N\left(I-D_{3} N\right)^{-1} C_{1} & B\left(I-N D_{3}\right)^{-1} M \\
L\left(I-D_{3} N\right)^{-1} C_{1} & K+L\left(I-D_{3} N\right)^{-1} D_{3} M
\end{array}\right) .
$$

We require that the interconnection is $\mathbf{C}_{g}$-stable, i.e. $A_{\mathrm{e}}$ has all eigenvalues in $\mathbf{C}_{g}$. If the realizations for $\tilde{\Sigma}$ and $\Sigma_{F}$ are both $\mathbf{C}_{g}$-detectable and $\mathbf{C}_{g}$-stabilizable, then this is equivalent to the requirement that in the interconnection of Figure 1 the closed loop transfer matrices from $d, v_{1}, v_{2}$ to $u, y, z$ are all $\mathbf{C}_{g}$-stable, i.e. the rational matrices have all poles in $\mathbf{C}_{\boldsymbol{g}}$.

We can derive the following theorem:

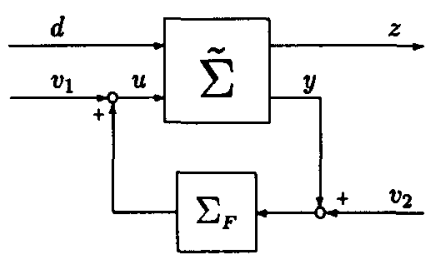

Fig. 1. 
Theorem 4.1. Let $\Sigma$ be given of the form (4.1). There exists a compensator of the form (2.2) such that the closed loop system is well-posed and $\mathbf{C}_{g}$-stable and such that the closed loop transfer matrix is equal to 0 if and only if the following conditions are satisfied:

(i) $(A, B)$ is $\mathbb{C}_{g}$-stabilizable, $\left(C_{1}, A\right)$ is $\mathbb{C}_{g}$-detectable,

(ii) $\left.\mathscr{T}_{g} \Sigma_{\mathrm{di}}\right) \subseteq \mathscr{V}_{g}\left(\Sigma_{\mathrm{ci}}\right)$

(iii) there exists a matrix $\tilde{N}$ such that

$$
\left(\left[\begin{array}{cc}
A & E \\
C_{2} & D_{4}
\end{array}\right]+\left[\begin{array}{c}
B \\
D_{2}
\end{array}\right] \tilde{N}\left[\begin{array}{ll}
C_{1} & D_{1}
\end{array}\right]\right)\left(\mathscr{T}_{g}\left(\Sigma_{\mathrm{di}}\right) \oplus \mathbb{R}^{q}\right) \subseteq\left(\mathscr{V}_{g}\left(\Sigma_{\mathrm{ci}}\right) \oplus\{0\}\right)
$$

and such that $I+\tilde{N} D_{3}$ is invertible.

$A$ controller solving the DDPMS is then described by

$$
\Sigma_{F}:\left\{\begin{array}{l}
\dot{p}=A p+B u+G\left(C_{1} p+D_{3} u-y\right) \\
u=F p-\tilde{N}\left(C_{1} p+D_{3} u-y\right)
\end{array}\right.
$$

Remarks. (1) Note that (4.4) describes a compensator of the form (2.2) because $I+\tilde{N} D_{3}$ is invertible. The reason for defining the compensator in this implicit way is to show the relationship with the compensator we found in (2.9).

(2) We can again express solvability of (4.3) in terms of subspace inclusions. However, the well-posedness constraint $\left(I+\tilde{N} D_{3}\right.$ is invertible) we can not express in subspace inclusions. On the other hand, condition (iii) is equivalent to the requirement that $\tilde{N}$ should satisfy a linear matrix equation and be such that $I+\tilde{N} D_{3}$ is invertible. Because all solutions to this linear matrix equation can be parametrized this is still a condition which can be checked straightforwardly.

We will only give a sketch of our proof. We will treat these extra feedthrough matrices in two steps. In the next subsection we show how we can reduce the disturbance decoupling problem with measurement and stability (DDPMS) for (4.1) to the same problem for a different system which has a direct feedthrough matrix from disturbance to output that is equal to zero. In the second subsection we show how we can reduce the DDPMS for a system (4.1) to the same problem but again for another system which this time has the form (2.1). On the latter system we may apply Theorem 2.2.

\subsection{A direct feedthrough matrix from disturbance to output}

We first solve DDPMS 'at infinity'. It is easily checked that for (4.1) the solvability of DDPMS 'at infinity' is equivalent to the existence of a matrix $S$ such that $I-S D_{3}$ is invertible and

$$
D_{4}+D_{2}\left(I-S D_{3}\right)^{-1} S D_{4}=0 .
$$

This fact is expressed in Theorem 4.1 by the condition that, in addition to $I+\tilde{N} D_{3}$ being invertible, the matrix $\tilde{N}$ has to be such that amongst others $D_{4}+D_{2} \tilde{N} D_{1}=0$. It is immediate that for such $\tilde{N}$ condition (4.5) can be satisfied by $S:=\left(I+\tilde{N} D_{3}\right)^{-1} \tilde{N}$.

Next we apply the preliminary feedback $u=S y+v$ to our system. The system we thus obtain will have a direct feedthrough matrix from $d$ to $z$ which will be equal to 0 . Clearly solvability of the disturbance decoupling problem for the original system is equivalent to solvability of the disturbance decoupling problem for the system we obtain after this preliminary feedback. Therefore we can reduce the disturbance decoupling problems for (4.1) to the same problems for a new system which has a direct feedthrough matrix from $d$ to $z$ that is equal to 0 .

\subsection{A direct feedthrough matrix from input to measurement}

By the previous subsection we may assume that we have system of the form (4.1) with $D_{4}=0$. Assume that a compensator $\Sigma_{F}$ of the form (2.2) is given such that the interconnection $\tilde{\Sigma} \times \Sigma_{F}$ is well-posed, 
$\mathbf{C}_{g}$-stable and has a closed loop transfer matrix which is equal to 0 . We define

$$
\begin{aligned}
\tilde{K} & :=K+L\left(I-D_{3} N\right)^{-1} D_{3} M, \\
\tilde{L} & :=L\left(I-D_{3} N\right)^{-1}, \\
\tilde{M} & :=\left(I-N D_{3}\right)^{-1} M, \\
\tilde{N} & :=N\left(I-D_{3} N\right)^{-1} .
\end{aligned}
$$

Then it is easily checked that the following compensator satisfies condition (i) of Theorem 2.2 for the system $\Sigma$ given by (2.1).

$$
\tilde{\Sigma}_{F}:\left\{\begin{array}{l}
\dot{p}=\tilde{K} p+\tilde{L} y, \\
u=\tilde{M} p+\tilde{N} y .
\end{array}\right.
$$

On the other hand assume that we have a compensator of the form (4.10) such that $I+D_{3} \tilde{N}$ is invertible and such that condition (i) of Theorem 2.2 is satisfied for $\Sigma$. In that case, the following compensator makes the interconnection $\tilde{\Sigma} \times \tilde{\Sigma}_{F}$ well-posed, $\mathbb{C}_{g}$-stable and yields a closed loop transfer matrix which is 0 :

$$
\begin{aligned}
K & :=\tilde{K}-\tilde{L}\left(I+D_{3} \tilde{N}\right)^{-1} D_{3} \tilde{M}, \\
L & :=\tilde{L}\left(I+D_{3} \tilde{N}\right)^{-1}, \\
M & :=\left(I+\tilde{N} D_{3}\right)^{-1} \tilde{M}, \\
N & :=\left(I+\tilde{N} D_{3}\right)^{-1} \tilde{N} .
\end{aligned}
$$

We can now apply Theorem 2.2 to $\Sigma$ described by (2.1) to obtain necessary and sufficient conditions for the solvability of DDPMS for $\Sigma$. We only have to do some work to incorporate the well-posedness constraint $\left(I+D_{3} \tilde{N}\right.$ invertible). The results of this subsection can be used to obtain necessary and sufficient conditions for the solvability of DDPMS of $\tilde{\Sigma}$ described by (4.1) with $D_{4}=0$. The results of the previous subsection can then be used to obtain necessary and sufficient conditions for the solvability of DDPMS for the general system $\tilde{\Sigma}$ of the form (4.1) without any restrictions. These conditions are given in Theorem 4.1.

\section{Conclusion}

In this paper we have treated the most general disturbance decoupling problem: the disturbance decoupling problem with measurement feedback and stability for some arbitrary stability set $\mathbf{C}_{\boldsymbol{g}}$. We have shown how the known results can be extended to incorporate direct feedthrough matrices in the system. In our opinion this paper completes the results already available.

\section{References}

[1] H. Akashi and H. Imai, Disturbance localization and output deadbeat control through an observer in discrete-time linear multivariable systems, IEEE Trans. Automat. Control 24 (1979) 621-627.

[2] G. Basile and G. Marro, Luoghi caratteristici dello spazio degli stati relativi al controllo dei sistemi lineari, L'Elettrotecnica 55 (12) (1968) 1-7.

[3] G. Basile and G. Marro, Controlled and conditioned invariant subspaces in linear system theory, J. Optim. Theory Appl. 3 (5) (1969) 306-315.

[4] M.L.J. Hautus and H.L. Trentelman, Linear Multivariable Systems, In preparation. 
[5] H. Imai and H. Akashi, Disturbance localization and pole shifting by dynamic compensation, IEEE Trans. Automat. Control 26 (1981) 226-235.

[6] A.S. Morse and W.M. Wonham, Decoupling and pole assignment by dynamic compensation, SIAM J. Control Optim. 8 (3) (1970) 317-337.

[7] J.M. Schumacher, Compensator synthesis using $(C, A, B)$ pairs, IEEE Trans. Automat. Control 25 (1980) $1133-1138$.

[8] J.M. Schumacher, Regulator synthesis using $(C, A, B)$ pairs, IEEE Trans. Automat. Control 27 (1982) 1211-1221.

[9] J.M. Schumacher, Dynamic Feedback in Finite and Infinite Dimensional Linear Systems, Math. Centre Tracts 143 (Amsterdam, 1981).

[10] A.A. Stoorvogel, The $H_{\infty}$ control problem: a state-space approach, Ph.D. Thesis, Eindhoven University of Technology, Netherlands (1990).

[11] A.A. Stoorvogel, The singular minimum entropy $H_{\infty}$ control problem, Systems Control Lett. 16(6) (1991) 411-422.

[12] A.S. Stoorvogel, The singular linear quadratic Gaussian control problem, COSOR Memorandum 90-43, Eindhoven University of Technology (1990); submitted for publication.

[13] A.A. Stoorvogel, A. Saberi and B.M. Chen, A reduced order observer based controller for $H_{\infty}$-optimization, Report (1991); submitted for publication in Systems Control Lett.

[14] H.L. Trentelman, Almost Invariant Subspaces and High Gain Feedback, CWI Tracts, Vol. 29 (Amsterdam, 1986).

[15] S. Weiland and J.C. Willems, Almost disturbance decoupling with internal stability, IEEE Trans. Automat. Control 34 (1989) 277-286.

[16] J.C. Willems, Least squares stationary control and the algebraic Riccati equation, IEEE Trans. Automat. Control 16 (1971) 621-634.

[17] J.C. Willems, The Analysis of Feedback Systems (MIT Press, Cambridge, MA, 1971).

[18] J.C. Willems and C. Commault, Disturbance decoupling by measurement feedback with stability or pole-placement, SIAM J. Control Optim. 19 (4) (1981) 490-504.

[19] J.C. Willems, Almost invariant subspaces: an approach to high gain feedback design-Part I: almost controlled invariant subspaces, IEEE Trans. Automat. Control 26 (1981) 235-252.

[20] J.C. Willems, Almost invariant subspaces: an approach to high gain feedback design-Part II: almost conditionally invariant subspaces, IEEE Trans. Automat. Control 27 (1982) 1071-1084.

[21] W.M. Wonham and A.S. Morse, Decoupling and pole assignment in linear multivariable systems: a geometric approach, SIAM J. Control Optim 8 (1) (1970) 1-18.

[22] W.M. Wonham, Linear Multivariable Control: A Geometric Approach, 3rd ed. (Springer-Verlag, New York, 1985).

[23] J.W. van der Woude, Almost disturbance decoupling by measurement feedback: a frequency domain analysis, IEEE Trans. Automat. Control 35 (1990) 570-573. 